Benjamin Bizjan, Alexander Kuznetsov, Andrej Jeromen, Edvard Govekar, Brane Širok, High-speed camera thermometry of laser droplet generation,

Applied Thermal Engineering,

Volume 110,

2017,

Pages 298-305,

ISSN 1359-4311,

(C) 2016. This manuscript version is made available under the CC-BY-NC-ND 4.0 license http://creativecommons.org/licenses/by-nc-nd/4.0/

Link to published article:

http://www.sciencedirect.com/science/article/pii/S1359431116315411

DOI: https://doi.org/10.1016/j.applthermaleng.2016.08.182 


\title{
High-speed camera thermometry of laser droplet generation
}

\section{*Benjamin Bizjan1, Alexander Kuznetsov1, Andrej Jeromen', Edvard Govekar', Brane Širok ${ }^{1}$}

\begin{abstract}
This paper presents a high-speed thermal imaging method using a visible light camera, with application to the laser droplet generation process (LDG). In the experiment, a nickel wire-end was exposed to a collimated laser beam, and the subsequent process of wire melting, pendant droplet formation and its detachment were recorded by a high speed camera. Instantaneous temperature fields of the metal surface were calculated from the imaging data and were characterized by a very good spatial and temporal resolution (200x400 pixels at 13837 frames per second). The droplet temperature could be accurately calculated between the melting point of nickel $\left(1455^{\circ} \mathrm{C}\right)$ and approximately $1950^{\circ} \mathrm{C}$, where image saturation started to occur. The remaining pendant droplet was shown to cool much more rapidly than the detached droplet, which is due to the heat conduction to the solid wire. Except for the time immediately after the droplet separation, the temperature distribution across the melt droplets was found to be quite uniform. Apart from the possibility of temperature field calculation, it was also demonstrated that the high-speed images of the LDG process can accurately capture contours and oscillation dynamics of melt droplets.
\end{abstract}

Keywords: Laser droplet generation, Temperature measurement, High-speed camera, Melting, Droplet detachment, Fluid dynamics

\section{Introduction}

Precise high-speed temperature measurements are very important in many technological processes characterized by high material velocities or short duration. An example of such a process is the laser droplet generation (LDG), which can be used for many different technological applications such as joining [1,2], 3D structuring [3], repair of worn metal surfaces, microforming and microcasting [4]. Due to the presence of multiple flow phases and the very high temperatures of melt droplets (often in excess of $2000^{\circ} \mathrm{C}$ ), only non-contact methods can be used to measure the temperature. Non-contact temperature measurements are most commonly performed by pyrometers or infrared cameras [5-9], but these devices have several operating limitations. Pyrometers are only capable of single-point temperature measurements, while the infrared cameras, though capable of measuring instantaneous temperature

\footnotetext{
${ }^{1}$ Faculty of Mechanical Engineering, University of Ljubljana, Aškerčeva 6, 1000 Ljubljana, Slovenia emails: benjamin.bizjan@fs.uni-lj.si, brane.sirok@fs.uni-lj.si, edvard.govekar@fs.uni-lj.si, alexander.kuznetsov@fs.uni-lj.si
} 
fields, are not well-suited for application in high-speed processes due to their limited spatial resolution and dynamic response as well as the high cost.

Since the LDG process is characterized by very high temperatures, the solid and liquid metal phases glow, emitting a high luminous flux. With that said, the temperature of the process can be measured by means of high-speed visible light cameras, which are characterized by much higher recording rates and image resolution than their infrared counterparts. The concept of using standard CCD and CMOS digital cameras for temperature measurement has attracted several authors so far, especially in the area of metallurgy (Chen et al. [10], Zhang et al [11], Panditrao and Rege [12]) and combustion studies (Jiang et al. [13], Kuhn et al. [14], Guo et al [15]). The studies [1215] employed color (RGB) cameras with the so-called two-color approach, where the ratio of image brightness in two different color channels was used to determine the temperature. Yan and Li [16] proposed and evaluated an algorithm and a system for two-color temperature measurement, while Ma and Zhang [17] discussed the limitations of the method due to the image saturation. A different approach was taken by Chen et al. [10], Zhang et al. [11] and Bizjan et al. [18,19] who used a measurement system with a monochrome camera.

There are certain advantages of the two-color method over the monochrome method, namely the lack of need for the reference point temperature measurement and a reduced measurement error in the case of a non-constant spectral emissivity [16]. On the other hand, the two-color method suffers from a more narrow temperature measurement range. Also, most of today's color cameras use a Bayer filter mosaic array on top of the imager, which effectively reduces both the image resolution and light sensitivity when compared to the monochrome camera of the same pixel count [11]. This becomes particularly problematic in high-speed applications where image resolution is already limited by high acquisition rates, and it is often difficult to provide sufficient lighting due to extremely short exposure times required for capturing sharp images. For these reasons, the monochrome visible-light cameras seem to be best suited for high speed temperature measurements. Using a monochrome high-speed camera, Bizjan et al. $[18,19]$ recorded the mineral wool fiberization process and was able to calculate temperature fields with a high spatial and temporal resolution, and a good accuracy. In the present paper, this method will be applied to the LDG process to accurately represent the typical thermal and hydrodynamic phenomena of this promising manufacturing process.

\section{Theoretical background and methodology}

Laser-molten nickel droplets can reach temperatures in excess of $2000^{\circ} \mathrm{C}$, meaning a substantial fraction of light is emitted in the part of the spectrum between $400 \mathrm{~nm}$ and $1000 \mathrm{~nm}$, where standard CCD and CMOS camera sensors are normally most sensitive for electromagnetic radiation. Due to a very short duration of the LDG process, highspeed visible light cameras are well suited to capture the process dynamics while achieving a good image resolution. 
In this manuscript, experimental temperature measurements were performed by a high-speed monochrome camera and a temperature calculation algorithm proposed in our previous publications $[18,19]$. This section will provide a brief overview of the method. The input information required for temperature calculation is the normalized image gray level 0 (black color) $\leq G \leq 1$ (white color), which can be assumed to be proportional to the camera sensor voltage response due to the incident light. The following set of equations was proposed for temperature calculation $[18,19]$ :

$$
\begin{aligned}
& T_{K}=C \sqrt[4]{\frac{G}{k \cdot \eta\left(T_{K}\right) \cdot t_{E} \cdot \sigma_{S}}}, \\
& \eta=\frac{\int_{0}^{\infty} Y(\lambda) B_{\lambda}(\lambda) d \lambda}{\int_{0}^{\infty} B_{\lambda}(\lambda) d \lambda} \approx \frac{\sum_{0.4 \mu m}^{1 \mu m} Y(\lambda) B_{\lambda}(\lambda) \Delta \lambda}{\frac{2}{15} \frac{\pi^{4} k_{B}{ }^{4}}{h^{3} c^{2}}}, \\
& B_{\lambda}=\frac{2 h c^{2}}{\lambda^{5}} \frac{1}{\exp \left(h c / \lambda k_{B} T_{K}\right)-1} .
\end{aligned}
$$

In Eqs. (1)-(3), $T_{\mathrm{K}}$ is the absolute temperature in [K], $k$ is the camera sensor sensitivity, $t_{\mathrm{E}}$ the camera shutter time, $\eta$ the light efficacy, $Y$ the sensor quantum efficiency, $\lambda$ the wavelength of light and $B_{\lambda}$ the spectral radiance. The physical constants are as follows: $\sigma_{\mathrm{S}}=5.67 \cdot 10^{-8} \mathrm{Wm}^{-2} \mathrm{~K}^{-4}$ (Stefan-Boltzmann constant), $k_{\mathrm{B}}=1.381 \cdot 10^{-23} \mathrm{~J} / \mathrm{K}$ (Boltzmann constant), $h=6.626 \cdot 10^{-34} \mathrm{~J} \cdot \mathrm{s}$ (Planck constant), $c=2.998 \cdot 10^{8} \mathrm{~m} / \mathrm{s}$ (speed of light in vacuum).

The constant $C$ depends on the measurement set-up, and implicitly contains all the variables which are not directly measured or known, but can be assumed to remain constant during the experiment. These variables include, but are not limited to, the surface emissivity $\varepsilon$ (proportional to $C^{4}$ ), lenses aperture setting, focal distance and internal light absorption. $C$ is obtained from Eq. (1) by calibration to a surface with a known reference temperature and the corresponding image gray level at that location. In our case, the reference temperature was measured by a pyrometer.

In Eq. (1), the glowing surfaces are treated as gray bodies, i.e. the emissivity is assumed to be constant with temperature and with wavelength of emitted light. Such assumption is justified due to relatively narrow wavelength ranges in which visible light cameras operate, and consequently also narrow temperature measurement ranges. Using the above-presented algorithm, the temperature fields can be obtained by element-wise transformation of image gray level matrices. 


\section{Experimental set-up}

The experimental set-up comprised the laser droplet generation (LDG) system and the temperature data acquisition system (Fig. 1).

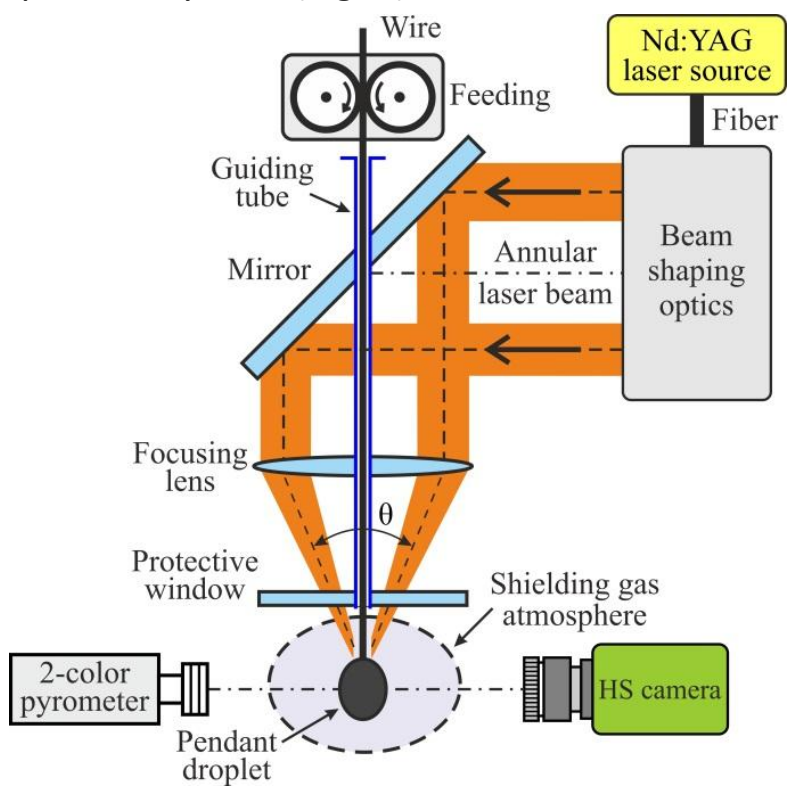

Fig. 1. Experimental set-up for laser droplet generation thermometry

The LDG system consists of a pulsed Nd:YAG laser source and an LDG head. The LDG head is used to shape a collimated beam into an annular ring, to guide the beam coaxially with the vertically fed metal wire and to focus the annular beam at the wireend along its circumference [6]. The LDG process runs in two sequential phases, i.e. a pendant droplet formation (Fig. 2a-2c) and pendant droplet detachment phase (Fig. $2 \mathrm{~d}-2 \mathrm{e}$ ). In the pendant droplet formation phase, a wire-end is melted by a pendant droplet formation pulse $P_{\mathrm{fp}}$ of lower power and longer duration. Under the action of surface tension and gravity force, a pendant droplet is formed from the molten wireend. During the pendant droplet formation, the wire must be fed to assure a proper position of the wire-end with respect to the annular laser beam focus. To detach the formed pendant droplet (diameter $D_{\text {pdr }}$ and temperature $T_{\text {pdr }}$ ), a high-power pulse $P_{\mathrm{d}}$ of shorter duration is applied. As a result, a detached droplet of diameter $D_{\mathrm{dr}}$ and temperature $T_{\mathrm{dr}}$ is generated with remaining pendant droplet of diameter $D_{\mathrm{rp}}$ and temperature $T_{\mathrm{rp}}$ staying attached to the wire-end. The detached droplet temperature $T_{\mathrm{dr}}$ is important from the point of view of its further application whereas the temperature $T_{\mathrm{rp}}$ of the remaining pendant droplet is of interest as an initial condition for generation of the next droplet. To characterize the related temperatures $T_{\mathrm{dr}}$ and $T_{\mathrm{rp}}$, a high speed camera and a two-wavelength pyrometer were utilized during the LDG process. 
Pendant droplet formation phase Droplet detachment phase



Fig. 2. Phases, parameters and outcomes of the laser droplet generation process

Temperature field measurements were carried out by a high-speed CMOS camera (Fastec HiSpec 4 mono 2G) with $85 \mathrm{~mm}$ lenses (f/16 aperture setting) and a $40 \mathrm{~mm}$ distance ring for macro photography. The camera sensor sensitivity was 1.524 lux $^{-1} \mathrm{~s}^{-1}$ and its quantum efficiency data is available in the product documentation [20]. Images were recorded with 13837 frames per second and had a resolution of $200 \times 400$ pixels, with a pixel size of $13.6 \mu \mathrm{m}$. The field of view was sized approximately $2.7 \mathrm{~mm} \times 5.4$ $\mathrm{mm}$. For comparison, FLIR images of the same process [5-9] only had a resolution of 32 x 64 pixels (pixel size $46 \mu \mathrm{m}$ ) and a frame rate of 1428 frames per second, despite covering a smaller field of view $(1.5 \mathrm{~mm} \times 3.0 \mathrm{~mm})$. Under such conditions, the useful temperature measurement range was between $1350^{\circ} \mathrm{C}$ and $1950^{\circ} \mathrm{C}$, with the accuracy of the method estimated to within $2 \%$ of the measurement range width [19].

Raw grayscale images obtained from the camera (sample image in Fig. 3a) were processed using presented temperature calculation algorithm (sample image in Fig. 3b). The upper droplet (i.e. pendant droplet) remains attached to the wire-end while the lower droplet (i.e. detached droplet) separates from the wire after the second (i.e. detachment) laser pulse. The temperature measurement method was calibrated to the melting point of nickel $\left(1455^{\circ} \mathrm{C}\right)$, at which the normalized image gray level temporarily stabilized $(G \approx 0.084$ ) due to the pendant droplet solidification. 


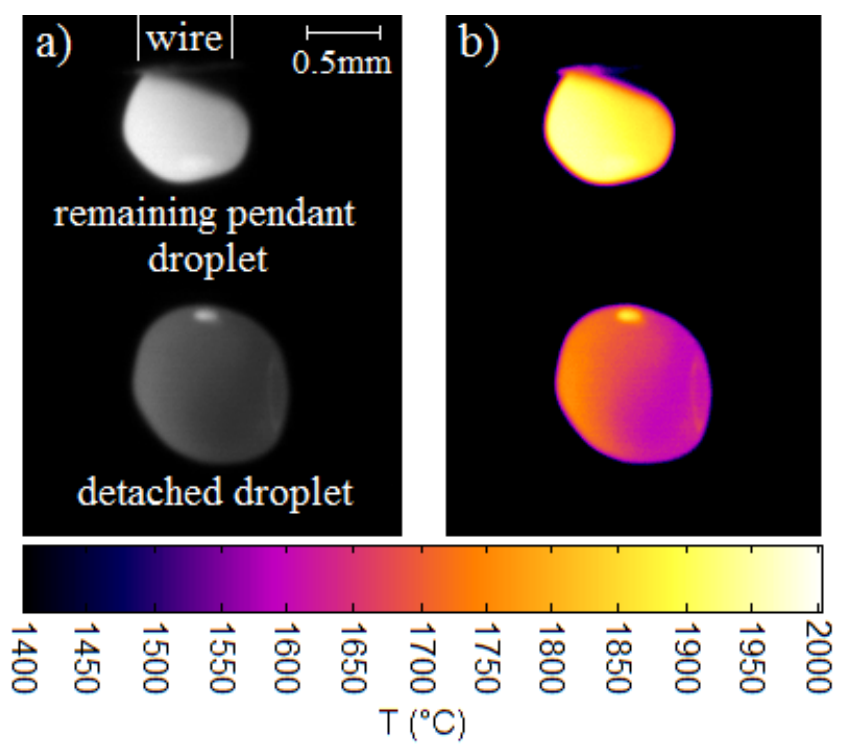

Fig. 3. A sample image of detached and remaining pendant droplets at $t=99.1 \mathrm{~ms}\left(t^{*}=\right.$ $4.1 \mathrm{~ms})$; a) raw camera image; b) calculated temperature field with the corresponding colormap

In addition to the temperature field measurements, point temperature readings were also obtained by a two-color pyrometer (Lascon LPCO3) of spectral wavelengths $\lambda_{1}=$ $1.65 \mu \mathrm{m}$ and $\lambda_{2}=2 \mu \mathrm{m}$. The pyrometer had a $3 \sigma$ measurement accuracy of $+-30 \mathrm{~K}$ in the temperature range from 500 to $3000{ }^{\circ} \mathrm{C}$, a time response of $0.2 \mathrm{~ms}$, and a minimum measuring spot diameter of $0.4 \mathrm{~mm}$. The calibration of the pyrometer was done with respect to the melting point of nickel. The pyrometer was focused to a fixed spot at the wire-end so that the temperature $T_{\mathrm{py}}$ captured during the pendant droplet formation and detachment phase corresponded to the pendant droplet temperature. After the detachment, the temperature $T_{\text {py }}$ corresponded to the temperature of the remaining pendant droplet. Readings in time intervals when the droplet was moved out of the pyrometer focus (due to the wire movement or melting) are of no particular importance for the analysis presented in this paper.

All of the measurements (in our experiment camera image acquisition, pyrometer readings, laser power output) were synchronized by a software trigger which was activated immediately before the first laser pulse. The moment of trigger activation defines the measurement time $t=0$.

\section{Results and discussions}

\subsection{Temperature analysis}

In the first step of experimental data processing, high-speed camera images were converted to thermal images (i.e. temperature fields) following the presented algorithm. In Fig. 4, the thermal images of the LDG process are shown at different times $\left(t_{1}\right.$ to $\left.t_{12}\right)$ after the triggering signal. 

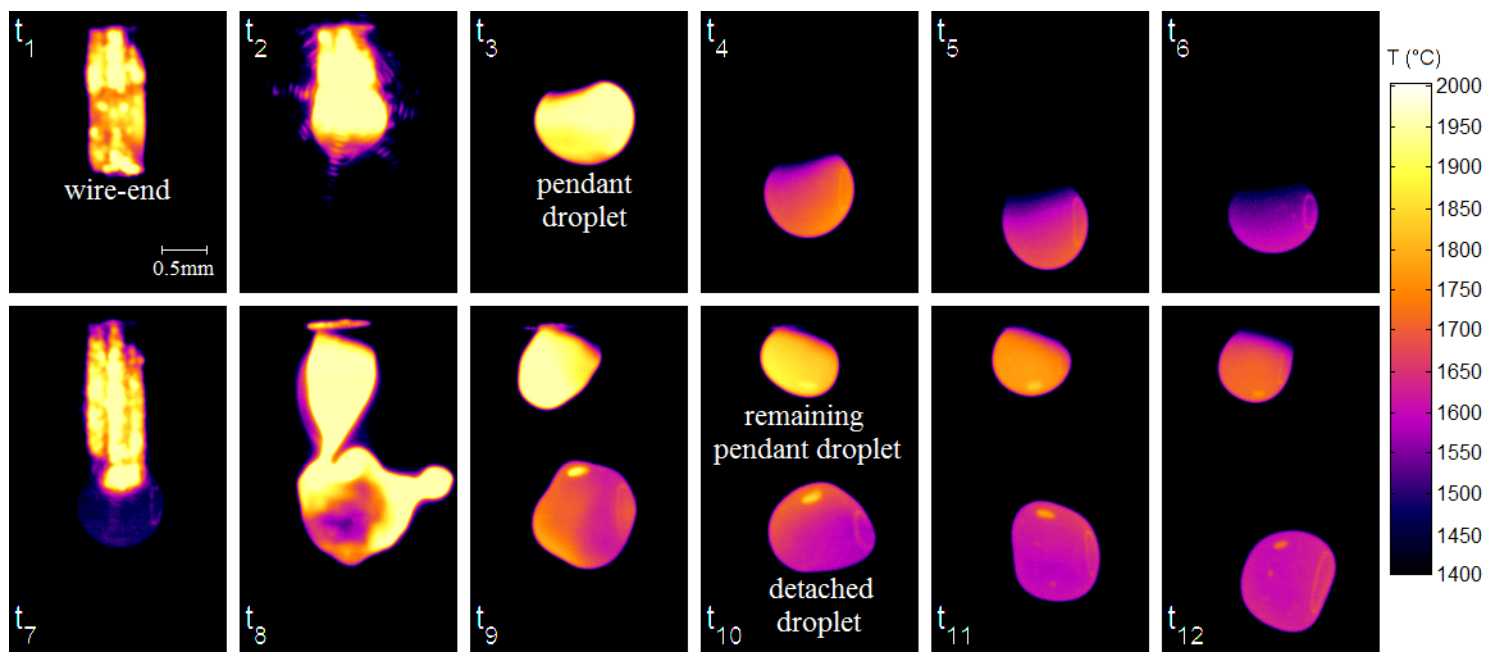

Fig. 4. Thermal images of the laser droplet generation process. Upper panel: $t_{1}=8.2$ $\mathrm{ms}$, frame separation $4 \mathrm{~ms}$; Lower panel: $t_{7}=93 \mathrm{~ms}$, frame separation $2.5 \mathrm{~ms}$

The upper panel of Fig. 4 presents the pendant droplet formation. At $t_{1}$, the solid nickel wire-end was exposed to a laser pulse, its tip gradually melted and a pendant droplet was formed at $t_{3}$ due to the surface tension of the molten material. At $t_{1}$ and $t_{2}$, the images indicate temperatures of up to $2000^{\circ} \mathrm{C}$ despite the fact that the melting point of nickel is at $1455^{\circ} \mathrm{C}$. Excessively high temperature readings and local image saturation (particularly at $t_{2}$ ) were a consequence of partial laser beam reflection, which significantly increases the amount of incident light on the camera sensor. For $t_{3}$ $\leq t<t_{7}$, the laser was turned off and the formed pendant droplet was positioned for its detachment. During this time interval, all the light was emitted due to the thermal radiation, allowing our temperature algorithm to produce much more credible results. In this time interval, the formed pendant droplet can be seen to cool down rapidly towards its solidification temperature $\left(1455^{\circ} \mathrm{C}\right)$.

The detachment laser pulse begins at $t_{7}$, when once again the temperature measurement is affected by light reflection, yielding implausibly high temperature readouts (the shape of the wire and pendant droplet is well visible, though). At $t_{8}$ when the laser pulse has already finished, a droplet is observed to detach from the column of molten metal. Once the detachment is complete $\left(t>t_{8}\right)$, both the detached and the remaining pendant droplet undergo large amplitude oscillations, which are gradually damped by melt viscosity as droplets cool $[5,6]$. A small part of the original pendant droplet remains attached to the wire-end while the detached droplet accelerates due to the gravity force [6]. Related droplet temperature fields gradually become more uniform.

Temperature conditions in the process, namely on the surface of related pendant and detached droplets can be presented in both spatial and temporal domain. For instance, temperature profiles can be used to present results in both domains simultaneously. In Figs. 5 and 6, the surface temperature profiles across the center of detached and 
remaining pendant droplets are shown at different times after the detachment laser pulse, $t^{*}=t-95 \mathrm{~ms}$.

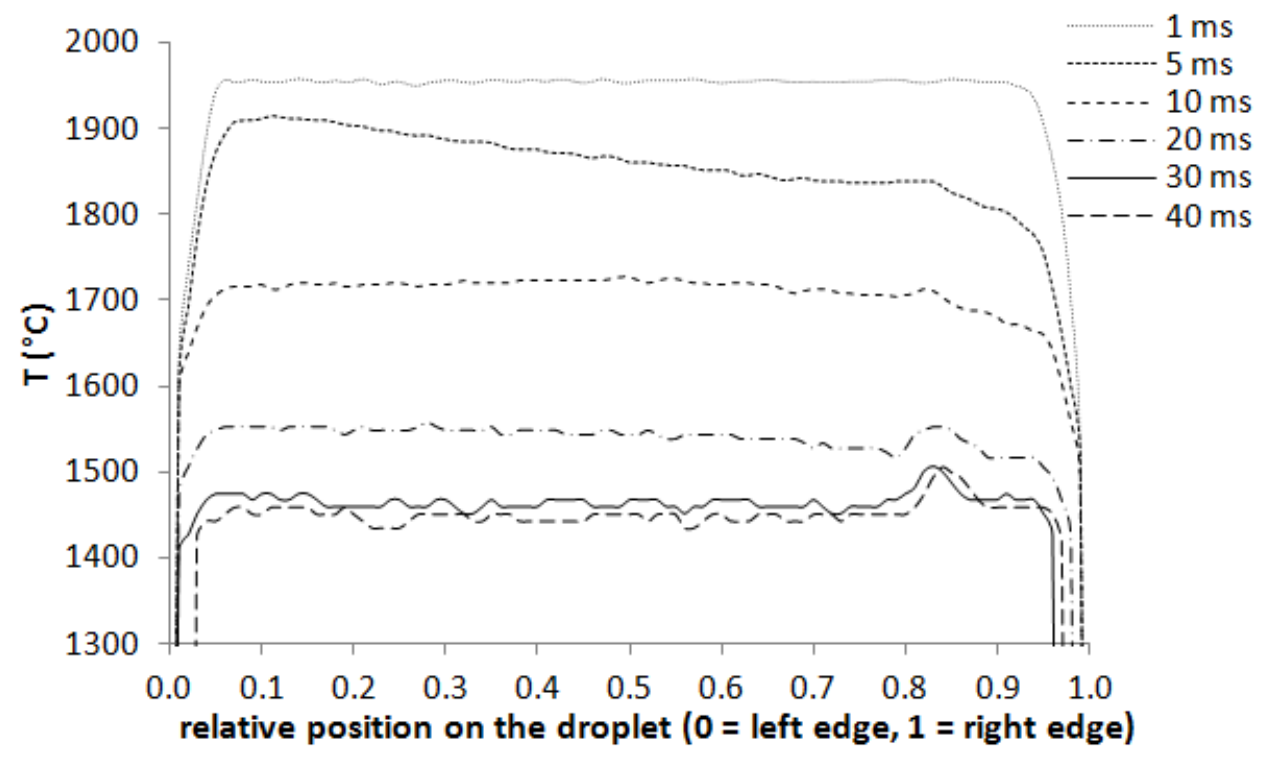

Fig. 5. Temperature profiles across the remaining pendant droplet at different times $t^{*}$

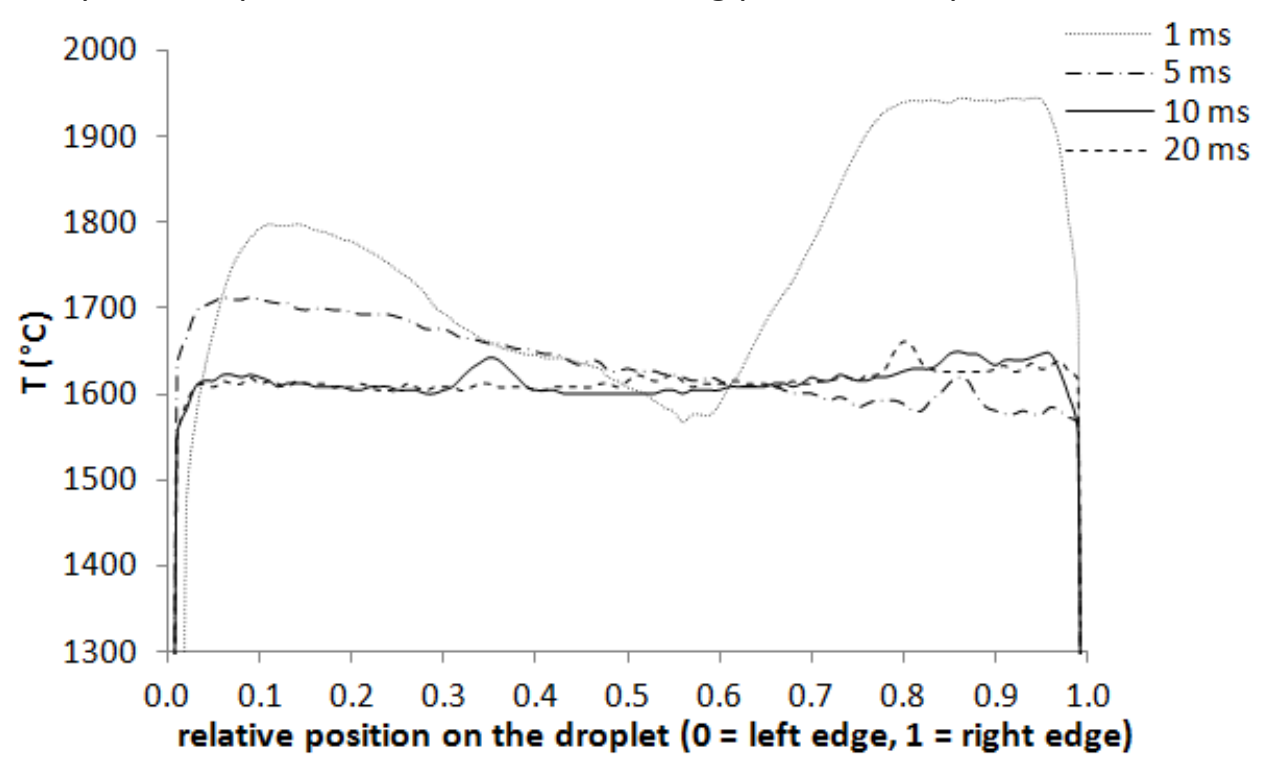

Fig. 6. Temperature profiles across the detached droplet at different times $t^{*}$

Regarding the temperature conditions immediately after the detachment laser pulse at $t^{*}=1 \mathrm{~ms}(t=94 \mathrm{~ms})$, it is evident that the detached droplet temperature is underestimated due to the image sensor saturation. As we will show in the following part of the manuscript, temperature readings by the pyrometer were well in excess of $2000^{\circ} \mathrm{C}$ while the temperatures in Figs. 5 and 6 only reach about $1950^{\circ} \mathrm{C}$. In the case of the remaining pendant droplet, saturation occurs across the almost complete droplet width. On the other hand, a much smaller area of the detached droplet was saturated at same $t^{*}$. 
At $t^{*}=5 \mathrm{~ms}$, the temperature steadily decreases by about $100 \mathrm{~K}$ between the left and right droplet edge of both droplets. This temperature gradient is related to the detachment laser pulse which, due to the asymmetric melt droplet pinch-off, resulted in the more intense droplet heating on its left side.

At times $t^{*} \geq 10 \mathrm{~ms}$, temperature profiles in Figs. 5 and 6 become increasingly uniform across the whole width of the droplet, with the exception of some anomalies caused by local light reflections (e.g. at relative position 0.85 in Fig. 5 and 0.35 in Fig. 6, when the temperature apparently increases by about $50 \mathrm{~K}$ ). The temperature of the remaining pendant droplet rapidly drops until about $t^{*}=30 \mathrm{~ms}$, when the melt begins to solidify. On the other hand, the detached droplet is only cooled slowly after its temperature field stabilizes at about $10 \mathrm{~ms}$, despite the fact that its temperature (1600 ${ }^{\circ} \mathrm{C}$ ) is still about $150 \mathrm{~K}$ above the melting point of nickel.

Considering the relatively low temperature gradients on the melt droplet surface, the temporal temperature characteristics with respect to the laser pulse $P(t)$ can be accurately represented by obtaining a time series of the mean droplet temperature, $T(t)$. In Fig. 7 , the laser pulse power $P=\left(P_{f}, P_{\mathrm{d}}\right)$ and related pyrometer-measured temperature $T_{\mathrm{py}}$ are plotted against the temperatures $T_{\mathrm{rp}}$ and $T_{\mathrm{dr}}$, which were calculated from the high-speed imaging data. Both the pyrometer and the temperature calculation method were calibrated to the melting point of nickel $\left(T=1455^{\circ} \mathrm{C}\right)$.

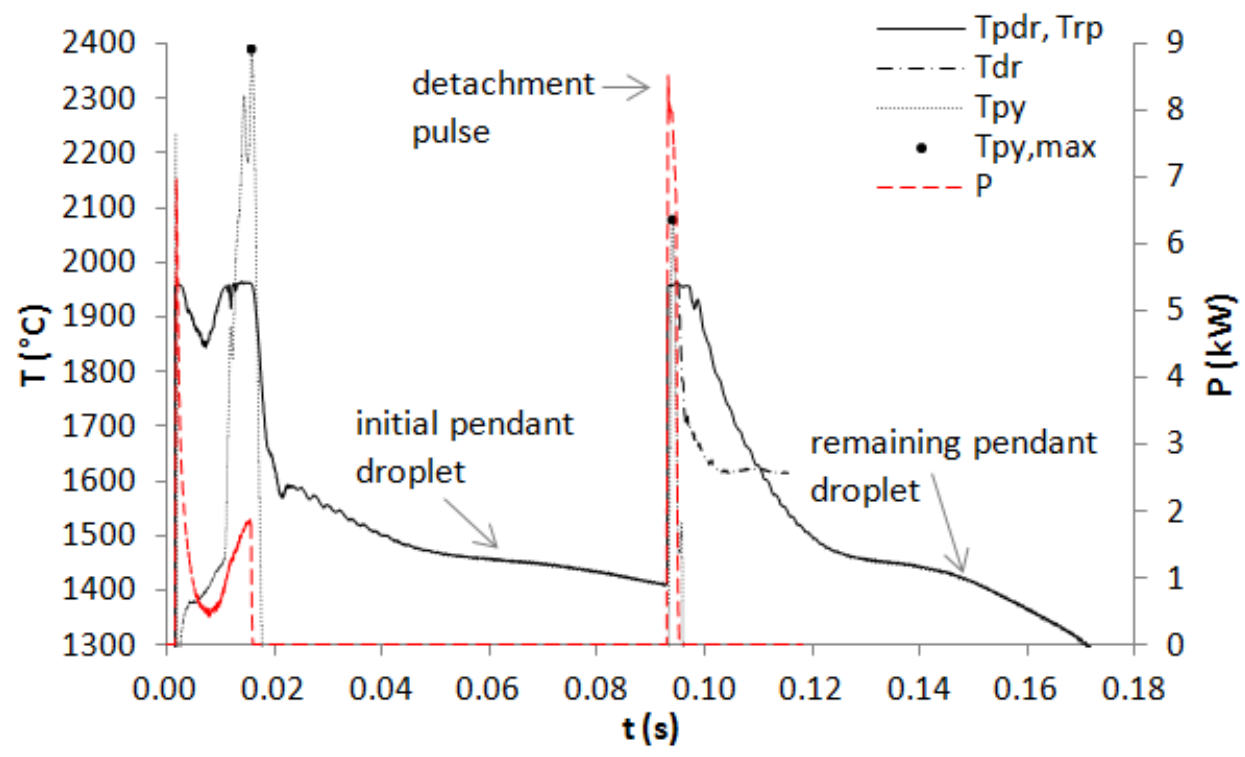

Fig. 7. Time series of the laser pulse power $P$ and related temperatures of detached and remaining pendant droplets

In Fig. 7, two melting events are clearly visible at the end of the pendant droplet formation pulse at approximately $t=0.02 \mathrm{~s}$ and at application of the detachment pulse at $t \approx 0.10 \mathrm{~s}$. After the end of each pulse, the resulting pendant droplets cool rapidly until the melt approaches its solidification temperature $\left(1455^{\circ} \mathrm{C}\right)$. As expected, the 
cooling rate is significantly reduced during the phase transition, but increases again once the droplet has solidified.

During the pendant droplet formation and the droplet detachment pulse, maximum pyrometer temperature readouts were $2392{ }^{\circ} \mathrm{C}$ and $2080{ }^{\circ} \mathrm{C}$, respectively. Note that the first peak of $T_{\text {py }}$ at $1.6 \mathrm{~ms}\left(2234^{\circ} \mathrm{C}\right)$ does not correspond to the actual wire surface temperature as the metal was still solid at that time, but is rather a consequence of laser beam reflection on metallic surface - the indicated temperature quickly decreases as laser power is reduced from its initial $7 \mathrm{~kW}$ peak. The quick drop of $T_{\mathrm{py}}$ after $\mathrm{t} \approx 17 \mathrm{~ms}$ is not only due to the cooling of the remaining pendant droplet, but also due to the wire feeding which shifts the pyrometer measurement spot to the solid wire above the remaining pendant droplet.

Laser beam reflections and large surface temperature ranges seem to be an even larger issue in the case of high-speed camera temperature measurements, as the camera sensor has a more narrow measurement range for incident light. Camera sensor reached saturation state (i.e. maximum gray level) at melt temperatures above approximately $1950{ }^{\circ} \mathrm{C}$. However, the camera-measured temperature curve correlates reasonably well to the pyrometer-measured temperature maxima. In order to reduce the light reflection effects and improve the droplet visibility in the pre-detachment phase, certain measures can be proposed. For example, a shortpass optical filter could be used to remove the problematic near-infrared wavelength range, where reflected laser beam light occurs. The presented temperature calculation algorithm could still be used by including the filter transmittance in the light efficacy equation (Eq. (2)).

Since the pendant droplet detachment is the most important phase of the droplet generation experiment, it will be a subject to additional detailed analysis. In Fig. 8, the temperature curves and the corresponding normalized gray level curves $G_{\mathrm{dr}}$ and $G_{\mathrm{rp}}$ are shown for detached droplet and remaining pendant droplet until the time when the detached droplet leaves the field of view.

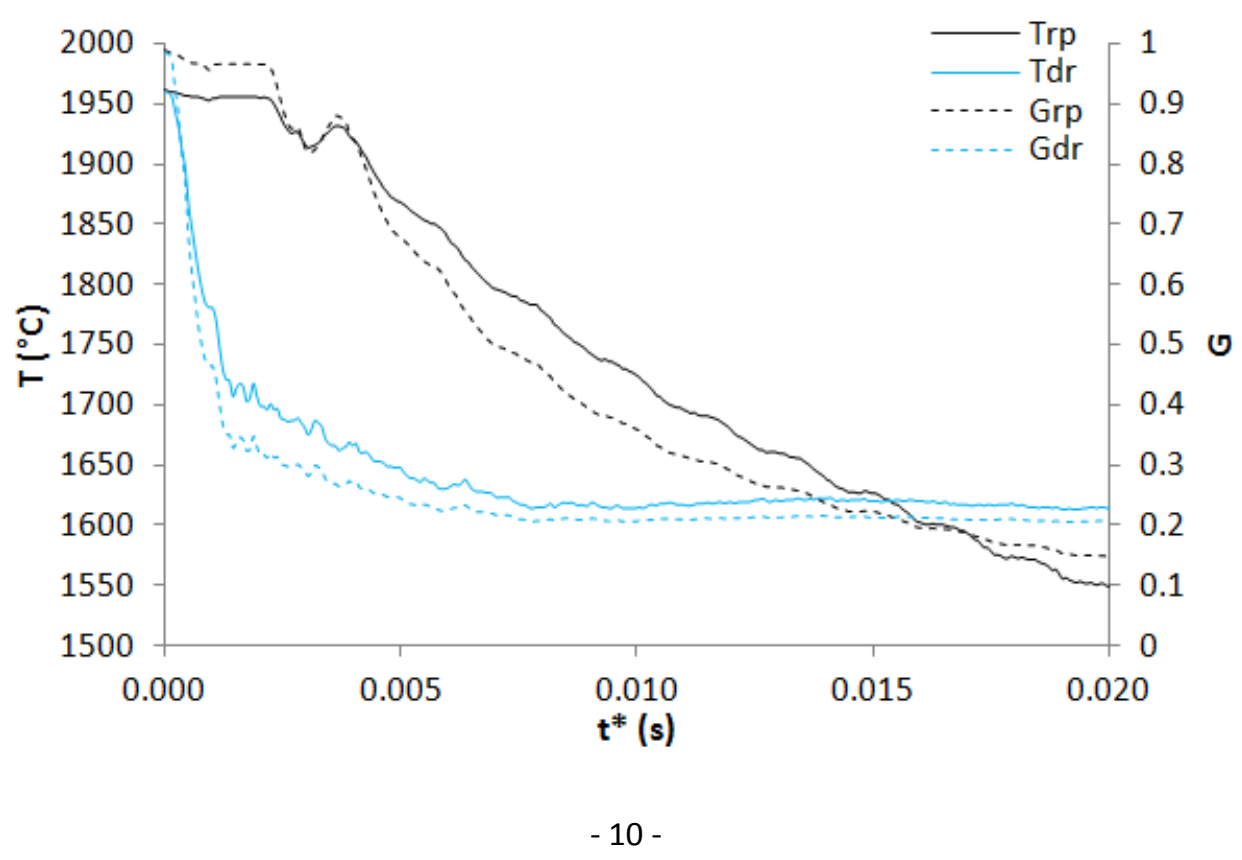


Fig. 8. Time series of droplet temperatures and normalized gray levels

As can be seen from Fig. 8, at $t^{*}=0.0 \mathrm{~ms}$ the related initial temperatures of both droplets are the same. Following the droplet separation at $t^{*}=0.6 \mathrm{~ms}$, temperature $T_{\mathrm{rp}}$ stagnates until $t^{*} \approx 2 \mathrm{~ms}$ and then drops at a rate of approximately $2.5 \cdot 10^{4} \mathrm{~K} / \mathrm{s}$. The remaining pendant droplet continues to cool quite rapidly due to the heat conduction to the wire to which it is attached.

On the other hand, the detached droplet temperature $T_{d r}$ initially starts to cool at a much higher rate $\left(2.5 \cdot 10^{5} \mathrm{~K} / \mathrm{s}\right)$, which is then reduced to approximately $1.5 \cdot 10^{4} \mathrm{~K} / \mathrm{s}$ in the time interval $1.5 \mathrm{~ms}<t^{*}<8 \mathrm{~ms}$. After $t^{*} \approx 8 \mathrm{~ms}$, the detached droplet maintains a nearly steady temperature until leaving the camera field of view. As the temperature of the detached droplet is still about $150 \mathrm{~K}$ above its solidification point, its low cooling rate is not a consequence of phase transition, but relatively slow heat transfer. Note that the detached droplet maintains a nearly steady position immediately after the detachment [6], reaching a velocity of about $0.2 \mathrm{~m} / \mathrm{s}$ at $t^{*}=20 \mathrm{~ms}$ due to the gravity acceleration. Therefore, the main heat transfer mechanism is by radiation and natural convection, and can be estimated to an order of magnitude of $10^{3} \mathrm{~K} / \mathrm{s}$.

\subsection{Image analysis by other methods}

As demonstrated, high-speed images of a process can be used to calculate the temperatures of glowing surfaces with a good spatial and temporal resolution. Nevertheless, such imaging data can also be analyzed by several other methods which do not involve temperature calculation, but may still provide valuable insight into the mechanism of observed process. Input grayscale images (sample image of detached droplet shown in Fig. 9a) can be converted to binary area images by gray level thresholding (Fig 9b), or to edge images (Fig. 9c) using an edge detection algorithm.
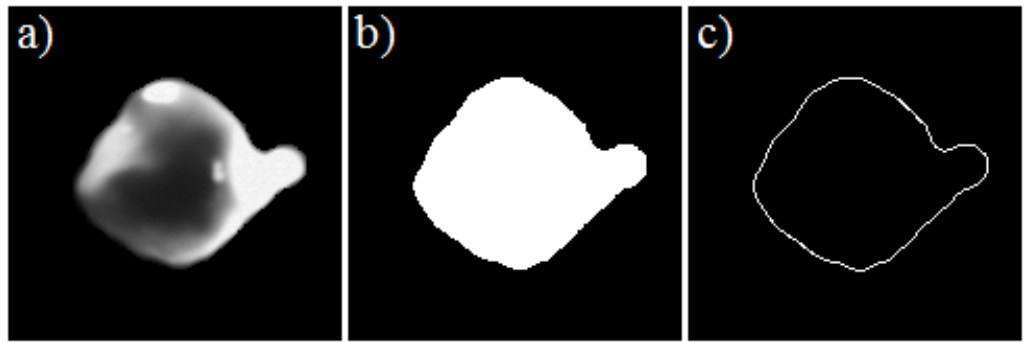

Fig. 9. a) Sample detached droplet image; b) binary area image; c) binary edge image

For the purpose of this paper, grayscale images were converted to binary area images using a threshold $G=0.1$, so that the areas with $G \geq 0.1$ were assigned the logical value 1 (white color) and the others the value 0 (black color). Then, the time series of droplet area $A$ was obtained as the total white area within the droplet image. Note that $A$ 
presents the area of droplet projection onto the plane of view, rather than the total surface area. The value of $A$ for the detached and remaining pendant droplet is shown in Fig. 10 for the time after the droplet separation.

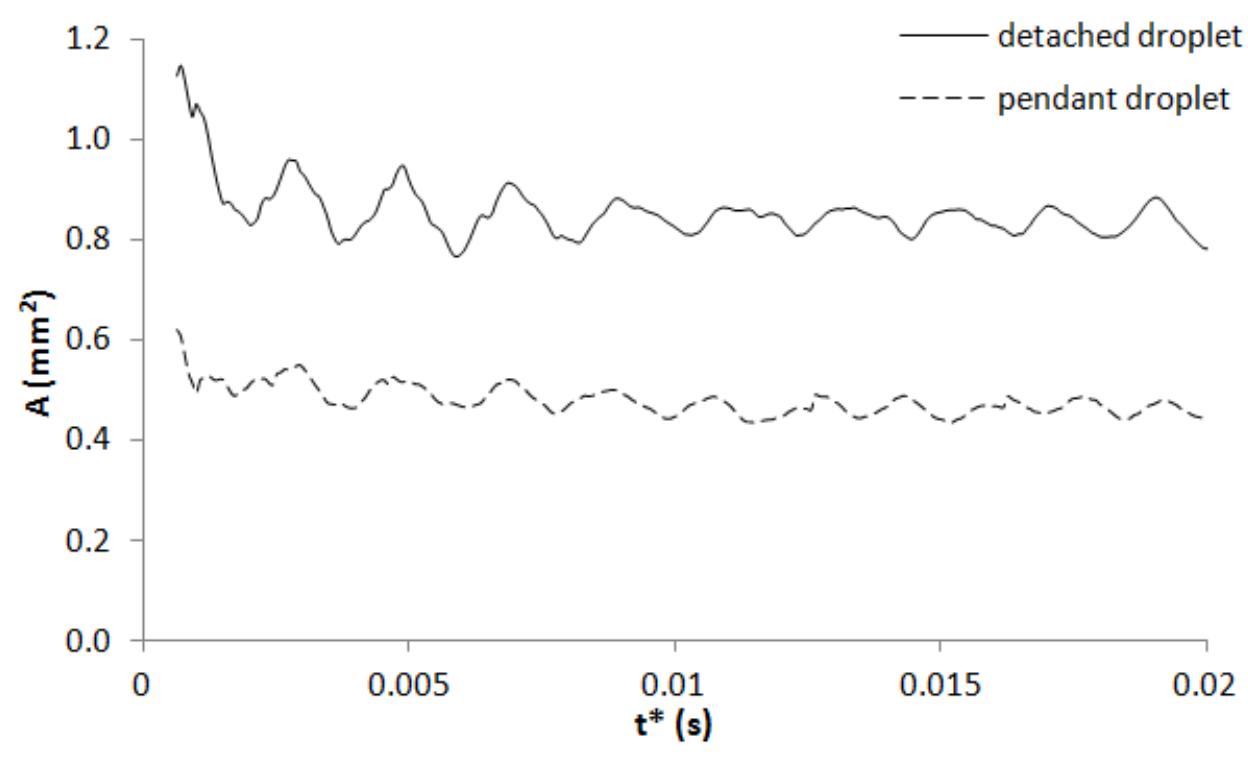

Fig. 10. Projection area of the detached and remaining pendant droplet

The maximum in droplet projection area occurred immediately after the droplet separation when both pendant and detached droplets experienced the most extreme deformation and eccentricity (Fig. 4). Both droplets experienced significant oscillations in the projection area which were gradually attenuated by the internal friction force due to the melt viscosity. The oscillations in $A$ were caused by the droplet natural oscillations as well as, in the case of the detached droplet, three-dimensional droplet rotation. The oscillation period of both detached and remaining pendant droplets can be estimated to $2.0 \mathrm{~ms}$, meaning the droplet natural frequency is approximately 500 $\mathrm{Hz}$.

Droplet oscillations can also be represented by droplet contours. In Fig. 11, the contours of the pendant and detached droplets are compared to their contours after one half of the oscillation period (i.e., $1.0 \mathrm{~ms}$ ). Although the droplet shape is asymmetric, the oscillation mode can clearly be identified as the second Rayleigh normal mode [5].

a)

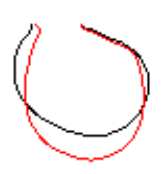

b)

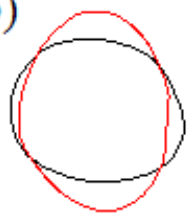


Fig. 11. Droplet contours at $t^{*}=4.8 \mathrm{~ms}$ (red line - gray in printed manuscript) and $t^{*}=$ $5.8 \mathrm{~ms}$ (black line); a) remaining pendant droplet b) detached droplet

\section{Conclusions}

In this paper, a high-speed thermal imaging method was applied to the analysis and characterization of a laser droplet generation process. The temperature fields measured by presented experimental set-up are characterized by an excellent spatial and temporal resolution, which has proven to be sufficient for accurate monitoring of heat transfer and hydrodynamic phenomena of the LDG process. Thermal imaging results indicate a large difference in the cooling rate between the detached droplet and the remaining pendant droplet, which cools more rapidly due to the heat conduction through the adjacent metal wire. Due to the slower cooling rate, the temperature of the detached droplet stays above the melting point, making the droplet applicable for various applications where a bond between the droplet and deposition spot is required. Apart from the temperature field calculation, the captured high-speed images also allowed to identify the oscillation modes of the droplets. With that said, the presented visible-light thermal imaging set-up may be more suitable and cost-effective for analysis of the LDG process than the ones employing infrared cameras, which are much more limited in high-speed capabilities. Further improvements of presented thermal imaging method can be achieved by reducing the laser beam reflection into the visualization optics. A shortpass optical filter could be applied to remove undesired laser beam reflections while retaining a large part of the thermally irradiated light. This way, the thermal imaging of the LDG process could be significantly improved in the melting phase prior to droplet detachment.

\section{Nomenclature}

$\begin{array}{ll}A & \text { droplet projection area, } \mathrm{m}^{2} \\ B_{\lambda} & \text { spectral radiance, } \mathrm{W} \cdot \mathrm{sr}^{-1} \cdot \mathrm{m}^{-3} \\ C & \text { calibration constant, }- \\ C & \text { speed of light, } \mathrm{m} \cdot \mathrm{s}^{-1} \\ D_{\mathrm{pdr}} & \text { pendant droplet diameter, } \mathrm{m} \\ D_{\mathrm{dr}} & \text { detached droplet diameter, } \mathrm{m} \\ D_{\mathrm{pdr}} & \text { remaining pendant droplet diameter, } \mathrm{m} \\ G & \text { normalized image gray level, }- \\ h & \text { Planck constant, } \mathrm{J} \cdot \mathrm{K} \\ K & \text { camera sensor sensitivity, lux }{ }^{-1} \cdot \mathrm{s}^{-1} \\ K_{\mathrm{B}} & \text { Boltzmann constant, } \mathrm{J} \cdot \mathrm{K}^{-1} \\ P & \text { laser power output, } \mathrm{W} \\ T & \text { temperature, }{ }^{\circ} \mathrm{C} \\ T_{\mathrm{K}} & \text { absolute temperature, } \mathrm{K} \\ T_{\mathrm{py}} & \text { pyrometer-measured temperature, }{ }^{\circ} \mathrm{C}\end{array}$




$\begin{array}{ll}t & \text { time since the trigger activation, } \mathrm{s} \\ t^{*} & \text { time since the detachment pulse, } \mathrm{s} \\ t_{\mathrm{E}} & \text { camera exposure time, } \mathrm{s} \\ Y & \text { camera sensor quantum efficiency, }- \\ \varepsilon & \text { emissivity, }- \\ \lambda & \text { wavelength of emitted light, } \mathrm{m} \\ \Delta \lambda & \text { discrete wavelength step for integration, } \mathrm{m} \\ \sigma_{\mathrm{S}} & \text { Stefan-Boltzmann constant, } \mathrm{W} \cdot \mathrm{m}^{-2} \cdot \mathrm{K}^{-4}\end{array}$

\section{References}

[1] Dreizin E.L. (1997) Droplet Welding: A New Technique for Welding Electrical Contacts. Welding Journal, vol. 4, p. 67-73.

[2] Conway P.P. (2002) Precision High Temperature Lead Free Solder Interconnections by Means of High-Energy Droplet Deposition Techniques. Annals of the CIRP, vol .51, no. 1, p. 177-180.

[3] Qi L.H., Chao Y.P., Luo J., Zhou J.M., Hou X.H., Li H.J. (2012) Novel Selection Method of Scanning Step for Fabricating Metal Components Based on Micro-Droplet Deposition Manufacture. International Journal of Machine Tools and Manufacture, vol. 56, p. 50-58.

[4] Zarzalejo L.J., Schmaltz K.S., Amon C.H. (1999) Molten Droplet Solidification and Substrate Remelting in Microcasting Part I: Numerical Modeling and Experimental. International Journal of Heat and Mass Transfer, vol. 34, p. 477-485.

[5] Kuznetsov A., Jeromen A., Govekar E. (2014) Droplet detachment regimes in annular laser beam droplet generation from a metal wire. CIRP Annals Manufacturing technology, vol. 63, p. 225-228.

[6] Govekar E., Kuznetsov A., Jerič A. (2016) Drop on demand generation from a metal wire by means of an annular laser beam. Journal of materials processing technology, vol. 227, p. 59-70.

[7] Krese B., Perc M., Govekar E. (2010) The dynamics of laser droplet generation. Chaos, vol. 20, article no. 013129.

[8] Krese B., Govekar E. (2011) Recurrence quantification analysis of intermittent spontaneous to forced dripping transition in laser droplet generation. Chaos, Solitons \& Fractals, vol. 44, p. 298-305.

[9] Krese B., Govekar E. (2011) Nonlinear analysis of laser droplet generation by means of 0-1 test for chaos. Nonlinear dynamics, vol. 67, no. 3, p. 2101-2109.

[10] Chen J., Osborn P., Paton A., Wall P. CCD near infrared temperature imaging in the steel industry. In: Instrumentation and Measurement Technology Conference, 1993. IMTC/93. Conference Record., IEEE, Irvine, CA, 1993, p. 299-303.

[11] Zhang Y., Xie Z., Hu Z., Zhao S., Bai H. (2014) Online Surface Temperature Measurement of Billets in Secondary Cooling Zone End-Piece Based on Data Fusion. IEEE transactions on instrumentation and measurement, vol. 63, no. 3, p. 612-619. 
[12] Panditrao A.M., Rege P.P. (2009) Estimation of furnace temperature distribution using digital photographic images. Association of Metallurgical Engineers of Serbia, vol. 15 , no. 2 , p. 115-123.

[13] Jiang Z.W., Luo Z.X., Zhou H.C. (2009) A simple measurement method of temperature and emissivity of coal-fired flames from visible radiation image and its application in a CFB boiler furnace. Fuel, vol. 88, p. 980-987.

[14] Kuhn P.B., Ma B., Connelly B.C., Smooke D.M., Long M.B. (2011) Soot and thinfilament pyrometry using a color digital camera. Proceedings of the Combustion Institute, vol. 33, no. 1, p. 743-750.

[15] Guo H., Castillo J.A., Sunderland P.B. (2013) Digital camera measurements of soot temperature and soot volume fraction in axisymmetric flames. Applied Optics, vol. 52, no. 33, p. 8040-8047.

[16] Yan J., Li W. (2010) Research on Colorimetric Temperature-measurement Method Improved Based on CCD Imaging. In: 3rd International Congress on Image and Signal Processing (CISP), Yantai, 2010, p. 189-192.

[17] Ma Z., Zhang Y. High Temperature Measurement Using Very High Shutter Speed to Avoid Image Saturation. AIP Conference Proceedings, vol. 1592, 2014, p. 246-253.

[18] Bizjan B., Širok B. Experimental investigation and modeling of mineral wool melt adhesion on a spinner wheel. In: B. ŠARLER (ed.), N. MASSAROTTI (ed.), P. NITHIARASU (ed.). Proceedings of the 3rd International Conference on Computational Methods for Thermal Problems, Bled, Slovenia, June 2-4 2014, p. 321-324.

[19] Bizjan B., Širok B., Drnovšek J., Pušnik I. (2015) Temperature measurement of mineral melt by means of a high speed-camera. Applied Optics, vol. 54, no. 32, p. 7978-7984.

[20] HiSpec Cameras Operator's Manual, Version 1.0, 2009 